\title{
Magneto-Hydrodynamic Squeeze Film Characteristics Between A Sphere and A Plane Surface
}

\author{
Tsu-Liang Chou \\ Associate Professor, Department of Mechanical Engineering, Nanya Institute of Technology, P.0. Box 324-22-59, \\ Chung-Li, Taiwan 320, R.O.C. \\ Jen-Wen Lai \\ Student, Department of Mechanical Engineering, Nanya Institute of Technology, P.O. Box 324-22-59, Chung-Li, Taiwan \\ 320, R.O.C. \\ Jaw-Ren Lin \\ Professor, Department of Mechanical Engineering, Nanya Institute of Technology, P.O. Box 324-22-59, Chung-Li, \\ Taiwan 320, R.O.C., jrlin@nanya.edu.tw
}

Follow this and additional works at: https://jmstt.ntou.edu.tw/journal

8 Part of the Mechanical Engineering Commons

\section{Recommended Citation}

Chou, Tsu-Liang; Lai, Jen-Wen; and Lin, Jaw-Ren (2003) "Magneto-Hydrodynamic Squeeze Film Characteristics Between A Sphere and A Plane Surface," Journal of Marine Science and Technology. Vol. 11: Iss. 3, Article 6. DOI: $10.51400 / 2709-6998.2281$

Available at: https://jmstt.ntou.edu.tw/journal/vol11/iss3/6

This Research Article is brought to you for free and open access by Journal of Marine Science and Technology. It has been accepted for inclusion in Journal of Marine Science and Technology by an authorized editor of Journal of Marine Science and Technology. 
Magneto-Hydrodynamic Squeeze Film Characteristics Between A Sphere and A Plane Surface

Acknowledgements

This paper is supported by the Natioal Science Council of Republic of China: NSC 92-2815-C-253- 004-E. 


\title{
MAGNETO-HYDRODYNAMIC SQUEEZE FILM CHARACTERISTICS BETWEEN A SPHERE AND A PLANE SURFACE
}

\author{
Tsu-Liang Chou*, Jen-Wen Lai**, and Jaw-Ren Lin***
}

Key words: squeezing films, electrically conducting fluids, magnetic fields, sphere, plane surface.

\begin{abstract}
Based upon the thin-film magneto-hydrodynamic (MHD) theory, this paper analyzes the squeeze-film characteristics between a sphere and plane surface lubricated with an electrically conducting fluid in the presence of a transverse magnetic field. The MHD Reynolds-type equation governing the squeeze-film pressure is derived using the continuity equation and the magneto-hydrodynamic motion equations. A closed-form solution for the squeezing film pressure is obtained, and applied to predict the MHD squeeze-film characteristics. According to the results obtained, the presence of externally applied magnetic fields signifies an enhancement in the squeeze-film pressure. On the whole, the magnetic-field effects characterized by the Hartmann number produce an increase in value of the load-carrying capacity and the response time as compared to the classical Newtonian-lubricant case. It improves the squeeze film characteristics of the sphere-plane surface system.
\end{abstract}

\section{INTRODUCTION}

Studies of squeezing-film characteristics play an important role in engineering science and industrial application such as skeletal joints, bio-lubrication, gears, bearings, machine elements, rolling elements and engine components. Traditionally, analyses of squeezefilm behavior focus upon the mechanism lubricated with a non-conducting viscous lubricant. Representative researches concerning with squeeze films have been presented for the rectangular plates by Hays [7], the parallel surfaces by Gould [4], the curved annular plates by Gupta and Vora [6], the spherical bodies by

Paper Submitted 10/03/03, Accepted 10/17/03. Author for Correspondence: Jaw-Ren Lin.

*Associate Professor, Department of Mechanical Engineering, Nanya Institute of Technology, P.O. Box 324-22-59, Chung-Li, Taiwan 320, R.O.C.

**Student, Department of Mechanical Engineering, Nanya Institute of Technology, P.O. Box 324-22-59, Chung-Li, Taiwan 320, R.O.C.

***Professor, Department of Mechanical Engineering, Nanya Institute of Technology, P.O. Box 324-22-59, Chung-Li, Taiwan 320, R.O.C.

E-mail: jrlin@nanya.edu.tw
Christensen [2], and the system between a sphere and a flat surface by Gould [5] and Conway and Lee [3]. With the development of modern machine elements, the use of an electrically conducting fluid as the lubricant to avoid the viscosity variation with temperature has been emphasized. By the use of an external magnetic field, a number of authors have successfully applied the electrically conducting lubricant to investigate various magneto-hydrodynamic (MHD) problems. Typical studies are found in the MHD journal bearings [8, 12], the MHD slider bearings $[1,10]$, the MHD squeeze-film annular disks [9] and the MHD squeeze-film rectangular plates [11]. Although the squeeze film action between a sphere and a flat surface lubricated with a non-conducting viscous lubricant has been detected [3,5], we have no idea how the magnetic-field effects affect this mechanism when lubricated with an electrically conducting fluid. For this reason, a further study is motivated.

In the present study, we are mainly concerned with the effects of externally applied magnetic fields on the squeeze film characteristics between a sphere and a flat plate with an electrically conducting fluid. Using the continuity equation and the magneto-hydrodynamic motion equations, the MHD Reynolds-type equation is derived and applied to predict the squeezing motion behavior. Comparing with the classical non-conducting-lubricant case, the results of squeeze film characteristics such as the load-carrying capacity and time-height relationship are presented for various values of Hartmann number.

\section{ANALYSIS}

Figure 1 shows the squeezing film geometry for the present study. A rigid sphere of radius is approaching a plane surface with a velocity under a constant load. The lubricant in the film region is taken to be an isothermal, incompressible electrically conducting fluid. An externally uniform transverse magnetic field $B_{0}$ is applied in the -direction. Under the usual thin-film lubrication theory, it is assumed that the fluid inertia is small, the body force is negligible except for the Lorentz 
force, and the induced magnetic field is small compared to the applied magnetic field. Based upon these assumptions, the continuity equation and the magnetohydrodynamic (MHD) momentum equations in polar coordinates reduce to the following.

$$
\begin{aligned}
& \frac{1}{r} \frac{\partial}{\partial r}(r u)+\frac{\partial w}{\partial z}=0, \\
& \frac{\partial p}{\partial r}=\mu \frac{\partial^{2} u}{\partial z^{2}}-\sigma B_{0}^{2} u, \\
& \frac{\partial p}{\partial z}=0,
\end{aligned}
$$

In the above equations, $u$ and $w$ denote the velocity components in the $r$ and $z$ directions respectively, $p$ is the film pressure, $\mu$ is the lubricant viscosity, and $\sigma$ is the electrical conductivity. The boundary conditions for the velocity components are:

$$
\begin{aligned}
& u=0, w=0 \text { at } z=0 \\
& u=0, w=\partial h / \partial t \text { at } z=h
\end{aligned}
$$

The lubricant film thickness, provided $R \gg r$, is given by:

$$
h=h_{m}+\frac{r^{2}}{2 R},
$$

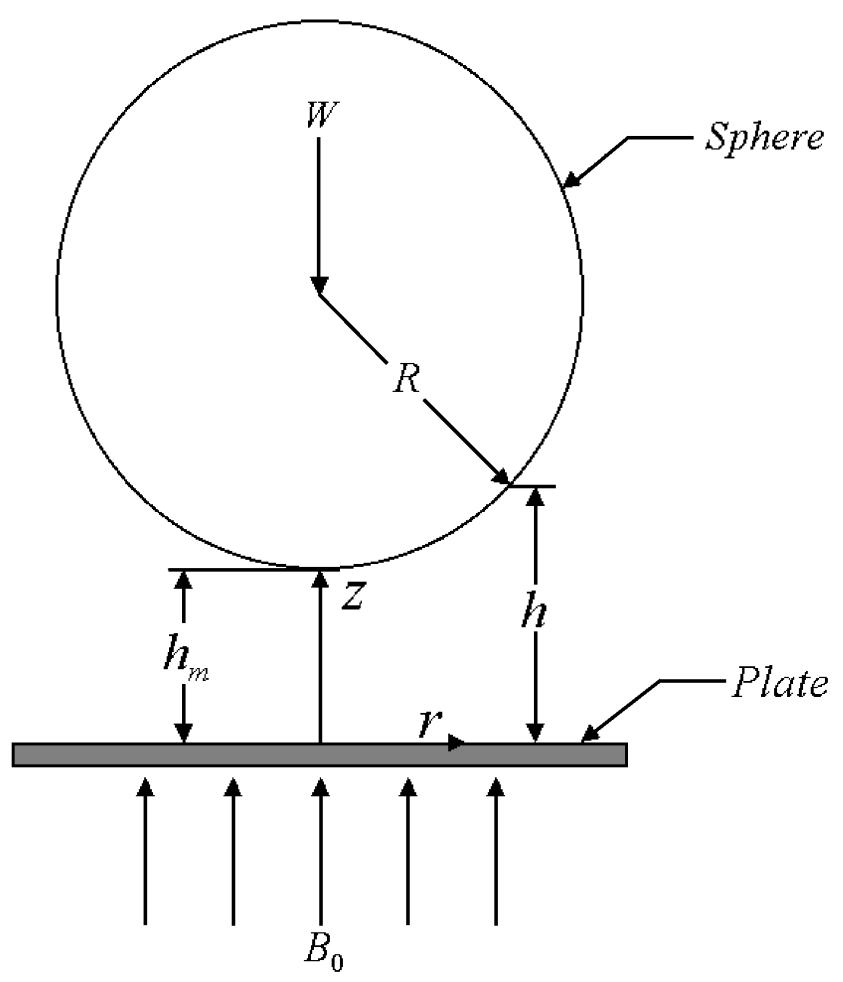

Fig. 1. Squeeze film geometry between a sphere and a plane surface in the presence of a transverse magnetic field. where $h_{m}$ represents the minimum film thickness at the central position. Integrating equation (2) with the above conditions one can obtain the velocity component $u$ :

$u=\frac{h_{m 0}^{2}}{\mu M^{2}} \frac{\partial p}{\partial r}\left\{\frac{\cosh \left(M h / h_{m 0}\right)-1}{\sinh \left(M h / h_{m 0}\right)} \sinh \left(\frac{M z}{h_{m 0}}\right)+1-\cos \left(\frac{M z}{h_{m 0}}\right)\right\}$.

In this equation, $h_{m 0}$ denotes the initial minimum film thickness and represents the Hartmann number defined by:

$$
M=B_{0} h_{m 0}\left(\frac{\sigma}{\mu}\right)^{1 / 2},
$$

Substituting the expression of $u$ into the continuity equation (1) and integrating the equation with respect to $z$ with the boundary conditions of $w$, one can obtain the magneto-hydrodynamic Reynolds-type equation governing the squeeze-film pressure.

$$
\frac{1}{r} \frac{\partial}{\partial r}\left\{\frac{h_{m 0}^{3}}{\mu M^{3}}\left[M \frac{h}{h_{m 0}}-2 \tanh \left(\frac{M h}{2 h_{m 0}}\right)\right] r \frac{\partial p}{\partial r}\right\}=\frac{\partial h}{\partial t} .
$$

This MHD Reynolds-type equation is applicable to the squeeze films between a sphere and a plane surface. As the value of $M$ approaches zero, this MHD Reynolds-type equation reduces to the non-conductinglubricant case $[3,5]$. Expressing in a non-dimensional form one has

$$
\frac{d}{d r^{*}}\left(F_{0}\left(h^{*}, r^{*}\right) r^{*} \frac{d p^{*}}{d r^{*}}\right)=-\frac{r^{*}}{\beta},
$$

where the dimensionless variables and parameters are:

$$
\begin{aligned}
& r^{*}=\frac{r}{R}, \quad h_{m}^{*}=\frac{h^{m}}{h_{m 0}}, \quad \beta=\frac{h_{m 0}}{R}, \quad p^{*}=\frac{p h_{m 0}^{2}}{\mu R\left(-d h_{m} / d t\right)}, \\
& F_{0}\left(h^{*}, M\right)=\frac{M h^{*}-2 \tanh \left(0.5 M h^{*}\right)}{M_{3}}, h^{*}=\frac{h}{h_{m 0}}=h_{m}^{*}+\frac{r^{* 2}}{2 \beta},
\end{aligned}
$$

For the squeeze-film problem considered, the pressure boundary conditions are:

$$
\begin{aligned}
& p^{*}=0 \text { at } r^{*}=1, \\
& \frac{d p^{*}}{d r^{*}}=0 \text { at } r^{*}=0 .
\end{aligned}
$$

Once the magneto-hydrodynamic squeeze-film pressure is known, the MHD squeeze-film characteris- 
tics can be evaluated.

\section{MHD SQUEEZE-FILM CHARACTERISTICS}

In engineering applications, the important magneto-hydrodynamic characteristics are the load-carrying capacity and the time-height relationship. Integrating the dimensionless magneto-hydrodynamic Reynoldstype equation (9) together with the boundary conditions (13) and (14), the MHD squeeze-film pressure is obtained.

$$
p^{*}=\frac{1}{2 \beta} \int_{r^{*}=r^{*}}^{r^{*}=1} \frac{r^{*}}{F_{0}\left(h^{*}, M\right)} d r^{*}
$$

The MHD load-carrying capacity is obtained by integrating the film pressure acting upon the sphere.

$$
W=\int_{r=0}^{\infty} 2 \pi r p d r .
$$

Expressing in a non-dimensional form and integrating the equation, the dimensionless load-carrying capacity is obtained.

$$
W^{*}=\frac{W h_{m 0}^{2}}{\mu R^{3}\left(-d h_{m} / d t\right)}=\frac{\pi}{\beta} \int_{r^{*}=0}^{1}\left\{\int_{r^{*}=r^{*}}^{r^{*}=1} \frac{r^{*}}{F_{0}\left(h^{*}, M\right)} d r^{*}\right\} r^{*} d r^{*} .
$$

For a steady applied load, we introduce the following dimensionless response time.

$$
t^{*}=\frac{W h_{m 0}^{2}}{\mu R^{4}} t
$$

The time-height relationship can be obtained from equation (17).

$$
\frac{d h_{m}^{*}}{d t^{*}}=-\frac{1}{\pi \int_{r^{*}=0}^{1}\left\{\int_{r^{*}=r^{*}}^{1}\left[r^{*} / F_{0}\left(h^{*}, M\right)\right] d r^{*}\right\} r^{*} d r} .
$$

Using the fourth-order Runge-Kutta method, the minimum film height at the central position can be numerically evaluated with the initial condition:

$$
h_{m}^{*}=1 \text { at } t^{*}=0 .
$$

\section{RESULTS AND DISCUSSION}

The MHD squeeze-film characteristics of a sphere and a plane surface lubricated with an electrically conducting fluid in the presence of a transverse magnetic field are analyzed in this study. With the aid of the definition of equation (8), the Hartmann number, $M$, signifies the effects of externally applied magnetic fields upon the magneto-hydrodynamic squeeze film characteristics. As the value of $M$ approaches zero, the MHD Reynolds-type equation reduces to the non-conducting-lubricant case. In the present analysis, results for the magneto-hydrodynamic squeeze-film pressure, the magneto-hydrodynamic load-carrying capacity and the response time are presented with: $\beta=0.05 ; M=$ $B_{0} h_{m 0}(\sigma / \mu)^{1 / 2}=0-5$.

The dimensionless film pressure $p^{*}$ generated by the squeeze film motion as a function of dimensionless coordinate $r^{*}$ for different values of Hartmann number $M$ at the minimum film thickness $h_{m}^{*}=0.3$ is presented in Figure 2. The black-circled line describes the nonconducting lubricant case. The others show the results of the system lubricated with an electrically conducting fluid in the presence of transverse magnetic fields. The influence of the applied magnetic field is visibly apparent. It is found the magnetic field effects result in a higher film pressure, especially in the vicinity of the position of minimum film height (i.e., the position at $r^{*}$ $=0$ ). Figure 3 shows the dimensionless maximum film pressure $p_{m}^{*}$ as a function of dimensionless minimum film height $h_{m}^{*}$ for different values of Hartmann number $M$. Compared with the non-conducting lubricant case, the magnetic field effects signify an increase in the value of $p_{m}^{*}$. In addition, the lower the minimum squeeze film height is achieved, the more the magnetic fields affect the maximum film pressure.

Figure 4 displays the dimensionless load-carrying capacity $W^{*}$ as a function of Hartmann number $M$ at

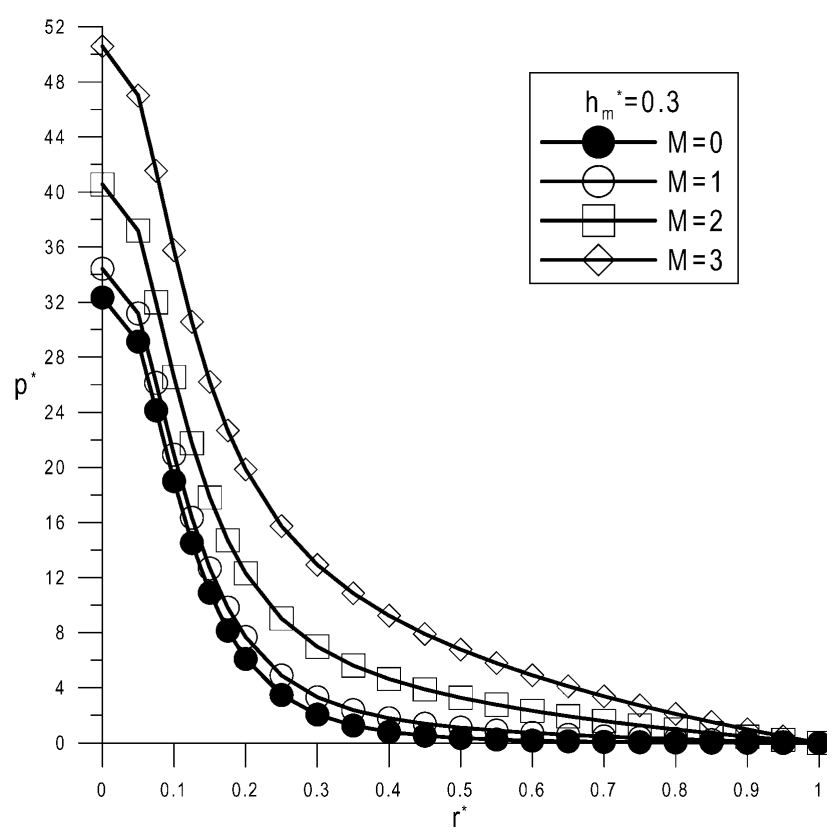

Fig. 2. Dimensionless film pressure as a function of dimensionless coordinate $r^{*}$ at $h_{m}^{*}=0.3$ for different values of $M$. 
various values of dimensionless minimum film height $h_{m}^{*}$. Since the magnetic field effects result in a higher film pressure, the integrated load-carrying capacity is similarly affected. As shown, the effects of magnetic fields produce an increase in the load-carrying capacity

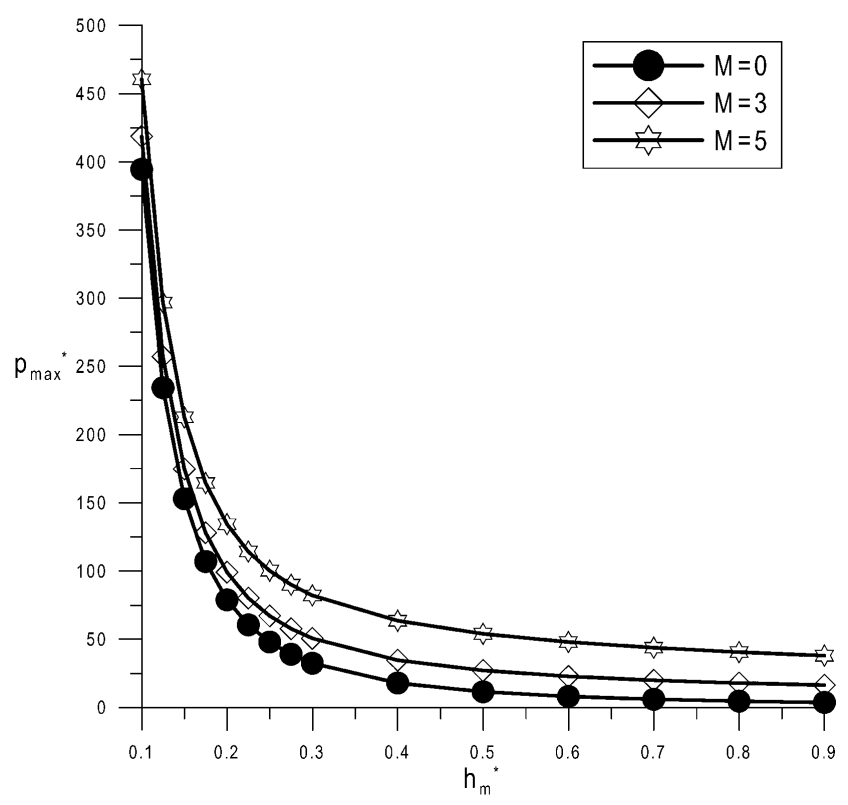

Fig. 3. Dimensionless maximum film pressure $p_{m}^{*}$ as a function of dimensionless minimum film thickness $h_{m}^{*}$ for different values of $M$.

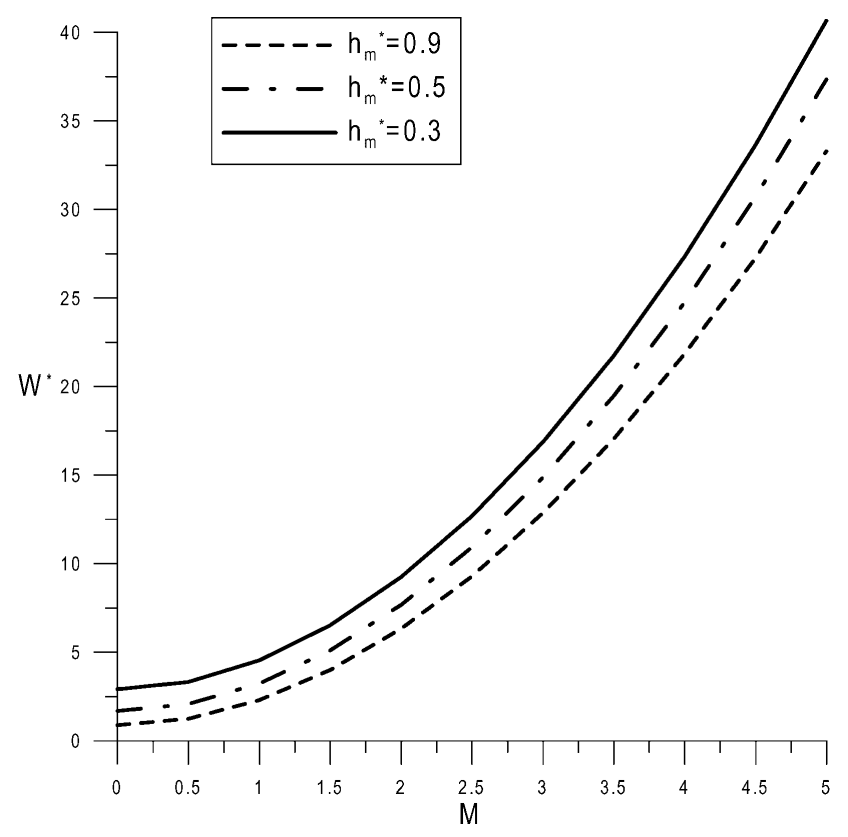

Fig. 4. Dimensionless load-carrying capacity $W^{*}$ as a function of Hartmann number $M$ at different values of $h_{m}^{*}$. with increasing Hartmann number. Figure 5 shows the dimensionless load-carrying capacity $W^{*}$ as a function of $h_{m}^{*}$ for different values of Hartmann number . Compared with the non-conducting lubricant case, the presence of magnetic fields increases the load-carrying capacity; and a larger increment is obtained with decreasing value of $h_{m}^{*}$ or increasing value of $M$. In general, the Hartmann number dominates the effects of magnetic fields upon the squeeze-film pressure and the load-carrying capacity. The effects of externally applied magnetic fields provide an enhancement in the load-carrying capacity, especially for small values of minimum film height.

Figure 6 represents the dimensionless response time $t^{*}$ as a function of the sphere displacement $1-h^{*}$ for different values of Hartmann number $M$. For the squeezing motion of the system, the initial condition is $h^{*}(0)=$ 1 , therefore the initial displacement is $1-h^{*}(0)=0$. At a same displacement, the effects of applied magnetic fields are observed to increase the value of response time. These phenomena can be realized that since the presence of the applied magnetic fields results in a higher load-carrying capacity, a longer response time would be required for the same distance to be achieved as compared to the non-conducting-lubricant case. As shown, the effects of applied magnetic fields upon the response time are more pronounced for larger values of $M$. Totally, the squeeze film characteristics between a sphere and a plane surface are improved by the use of an electrically conducting fluid in the presence of a transverse magnetic field.

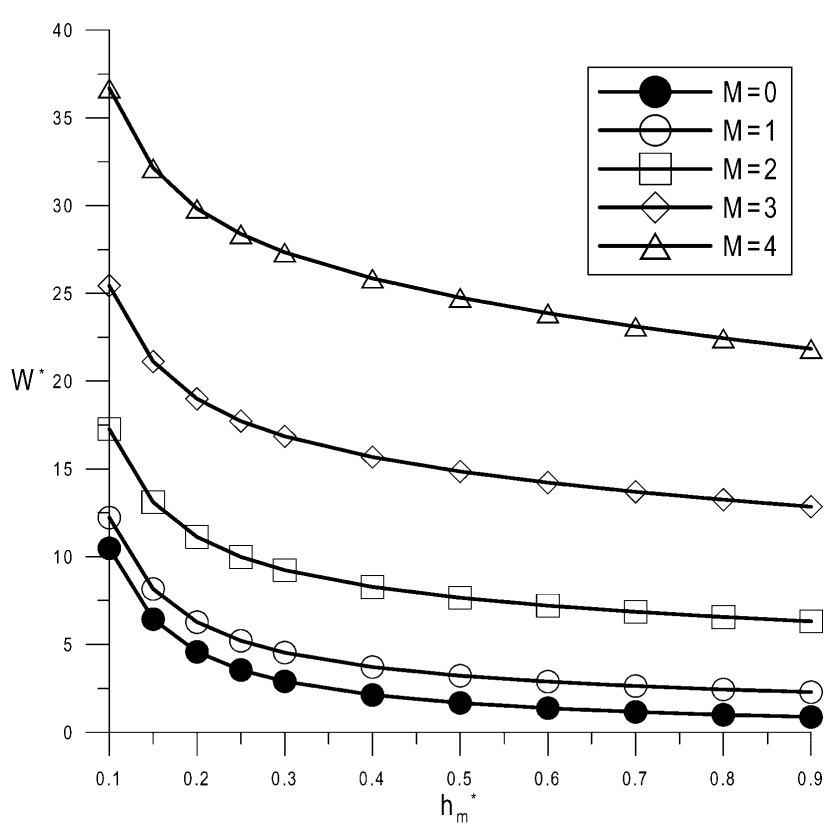

Fig. 5. Dimensionless load-carrying capacity $W^{*}$ as a function of minimum film thickness $h_{m}^{*}$ for different values of $M$. 


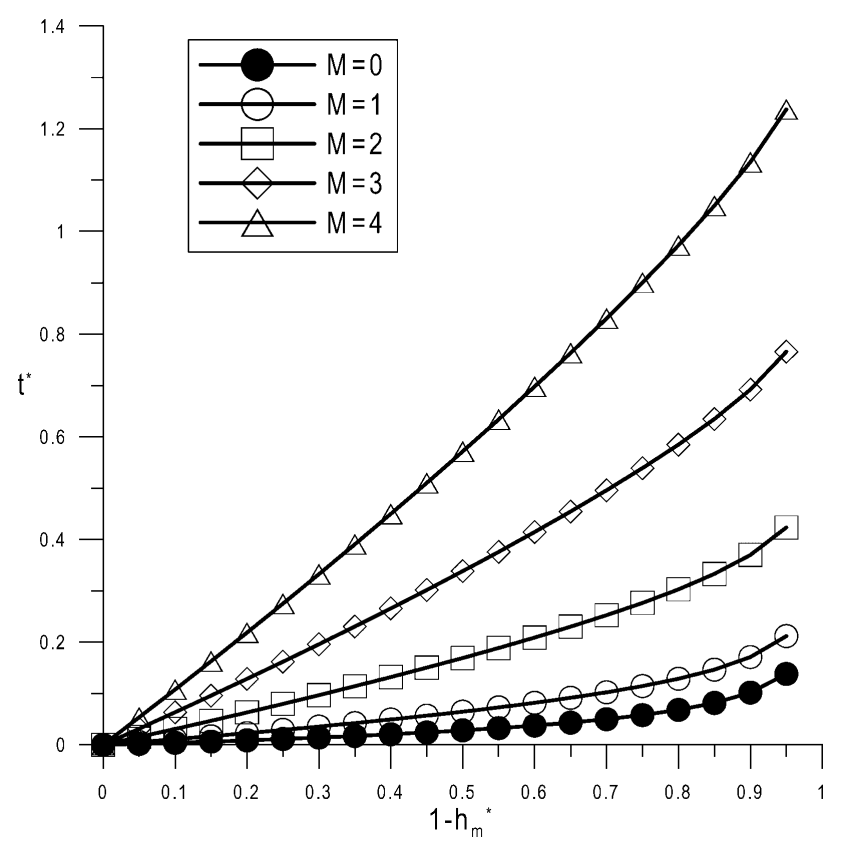

Fig. 6. Dimensionless response time $t^{*}$ as a function of sphere displacement $1-h^{*}$ for different values of $M$.

\section{CONCLUSIONS}

On the basis of the thin-film MHD theory, the magneto-hydrodynamic squeezing-film characteristics between a sphere and a plane surface lubricated with an electrically conducting fluid in the presence of a transverse magnetic field is presented. As the value of Hartmnn number approaches zero, the present study reduces to the non-conducting-lubricant case. According to the results obtained, conclusions can be drawn as follows.

We have derived the magneto-hydrodynamic Reynolds-type equation to analyze the magneto-hydrodynamic squeeze-film characteristics between a sphere and a plane surface lubricated with an electrically conducting fluid. It has been shown that the presence of externally applied magnetic fields signifies an increase in the MHD squeeze-film pressure. Comparing with the classical non-conducting-lubricant case, the effects of externally applied magnetic fields characterized by the Hartmann number provide an enhancement the MHD load-carrying capacity and lengthen the response time, especially for larger values of Hartmann number. On the whole, the squeeze film characteristics between a sphere and a plane surface are improved by the use of an electrically conducting fluid in the presence of a transverse magnetic field.

\section{ACKNOWLEDGEMENTS}

This paper is supported by the Natioal Science Council of Republic of China: NSC 92-2815-C-253004-E.

\section{REFERENCES}

1. Agrawal, V.K., "Inertia Effects in Hydromagnetic Inclined Slider Bearing," Jap. J. Appl. Physics, Vol. 9, pp. 820-824 (1970).

2. Christensen, H., "Elastohydrodynamic Theory of Spherical Bodies in Normal Approach," ASME J. Lubric. Technol., Vol. 92, pp. 145-154 (1970).

3. Conway, D. and Lee, H.C., "Impact of A Lubricated Surface by A Sphere," ASME J. Lubric. Technol., Vol. 97, pp. 613-615 (1975).

4. Gould, P., "Parallel Surface Squeeze Films," ASME J. Lubric. Technol., Vol. 89, pp. 375-380 (1967).

5. Gould, P., "High-pressure Spherical Squeeze Films," ASME J. Lubric. Technol., Vol. 93, pp. 207-208 (1971).

6. Gupta, J.L., and Vora, K.H., “Analysis of Squeeze Films Between Curved Annular Plates," ASME J. Lubric. Technol., Vol. 102, pp. 48-50 (1980).

7. Hays, D.F., "Squeeze Films for Rectangular Plates," ASME J. Basic Engin., Vol. 88, pp. 243-246 (1963).

8. Kuzma, D.C., "The Magnetohydrodynamic Journal Bearing," ASME J. Basic Engin., pp. 424-428 (1963).

9. Lin, J.R., "Magneto-hydrodynamic Squeeze Film Characteristics Between Annular Disks," Indust. Lubric. Tribol., Vol. 53, No. 2, pp. 66-71 (2001).

10. Lin, J.R., "Magneto-hydrodynamic Lubrication of Finite Slider Bearings," Int. J. Appl. Mechanics Engin., Vol. 7, No. 4, pp. 1229-1246 (2002).

11. Lin, J.R., "Magneto-hydrodynamic Squeeze Film Characteristics for Finite Rectangular Plates," Indust. Lubric. Tribol., Vol. 55, No. 2, pp. 84-89 (2003).

12. Malik, M., and Singh, D.V., "Analysis of Finite Magnetohydrodynamic Journal Bearings," Wear, Vol. 64, pp. 273-280 (1980). 